\title{
Approximation of Control for Singularly Perturbed System with Delay with Geometric Constraints
}

\section{V. Grebennikova, A. G. Kremlev}

Ural Federal University, 19, Mira st., 620002, Ekaterinburg, Russia, giv001@mail.ru, kremlev001@ mail.ru

The control problem for the singularly perturbed system with delay with indeterminate initial conditions and geometric constraints on the control resources according to the minimax criterion is considered. A limiting problem is formulated for which a specially selected quality functional is chosen. We propose the procedure for initial approximation construction of a control response in the control minimax problem.

Key words: singularly perturbed system with delay, optimal control, fundamental matrix

\section{References}

1. Krasovskii N. N. Teorija upravlenija dvizheniem [The Theory of Motion Control]. Moscow, Nauka, 1968, 475 p. (in Russian).

2. Kurzhanskij A. B. Upravlenie i nabljudenie v uslovijah neopredelennosti [Control and Surveillance in the Face of Uncertainty]. Moscow, Nauka, 1977, 392 p. (in Russian).

3. Kremlev A. G. Asymptotic properties of a set of trajectories of a singularly perturbed system in the optimal control problem. Autom. Remote Control [Avtomatika i Telemehanika], 1993, vol. 54, iss. 9, pp. 1353-1367.

4. Grebennikova I. V. Solution approximation in a minimax control problem for a singularly perturbed system with delay. Russian Math. [Izvestiya VUZ. Matematika], 2011, vol. 55, iss. 10, pp. 23-33. DOI: 10.3103/S1066369X11100045.

5. Grebennikova I. V. The problem of optimal control for singularly perturbed system with delay with integral quadratic constraints. Izv. Saratov Univ. (N.S.), Ser. Math. Mech. Inform., 2012, vol. 12, iss. 4, pp. 3-11 (in Russian).

6. Kremlev A. G., Grebennikova I. V. About asymptotic of a set of trajectories of a singularly perturbed system with delay. Novosti nauchnoj mysli - 2006: materialy mezhdunarodnoi nauch. prakt. konf. [News of Scientific Thought : proceedings of the international conference]. Dneproperovsk, Nauka i obrazovanie, 2006, vol. 4, pp. 65-69 (in Russian).

7. Rokafellar R. Vypuklyj analiz [Convex Analysis]. Moscow, Mir, 1973, 492 p. (in Russian).

8. Krasovskii N. N. Nekotorye zadachi teorii ustojchivosti dvizhenija [Some Problems in the Theory of Stability of Motion]. Moscow, Fizmatgiz, 1959, 468 p. (in Russian).

9. Kirillova F. M. Relative controllability of linear dynamic systems with delay. Doklady AN SSSR, 1967, vol. 174, iss. 6, pp. 1260-1263 (in Russian).

10. Bellman R., Kuk K. Differencial'no-raznostnye uravnenija [Differential-Difference Equations]. Moscow, Mir, 1967, 547 p. (in Russian).

11. Natanson I. P. Teorija funkcij veshhestvennoj peremennoj [Theory of Functions of a Real Variable]. Moscow, Nauka, 1974, 468 p. (in Russian).

УДК 514.174.3+519.65

\section{КОЭФФИЦИЕНТ ИЗОПЕРИМЕТРИЧНОСТИ СИМПЛЕКСА В ЗАДАЧЕ АППРОКСИМАЦИИ ПРОИЗВОДНЫХ}

\section{В. А. Клячин ${ }^{1}$, Д. В. Шуркаева ${ }^{2}$}

\begin{abstract}
${ }^{1}$ Доктор физико-математических наук, доцент кафредры компьютерных наук и экспериментальной математики, Волгоградский государственный университет, klchnv@ mail.ru

${ }^{2}$ Аспирант касредры компьютерных наук и экспериментальной математики, Волгоградский государственный университет, diana-547@ya.ru
\end{abstract}

В статье вводится величина $\sigma(G)=|\partial G|^{n /(n-1)} /|G|$ коэсрфициента изопериметричности области $G \subset \mathbb{R}^{n}$. В терминах этой величины получены оценки погрешности $\delta_{\Delta}(f)$ вычисления градиента при кусочно-линейной интерполяции фрункций классов $C^{1}(G), C^{2}(G), C^{1, \alpha}(G), 0<\alpha<1$. Задача получения таких оценок нетривиальна, особенно в многомерном случае. Здесь надо отметить, что в двумерном случае для фрункций класса $C^{2}(G)$ сходимость производных обеспечивается классическим условием Делоне. В многомерном же случае, как показывают примеры, подобного условия не достаточно. Тем не менее в статье показано, как применить полученные оценки для триангуляции Делоне многомерных дискретных $\varepsilon$-сетей. Полученные результаты дают достаточные условия сходимости производных на триангуляциях Делоне дискретных $\varepsilon$-сетей при $\varepsilon \rightarrow 0$. Кроме этого найдены соотношения искажения коэсрфицциента изопериметричности симплексов при квазиизометричном преобразовании.

Ключевые слова: коэсрсрициент изопериметричности, симплекс, кусочно-линейная интерполяция. 


\section{ВВЕДЕНИЕ}

Пусть $p_{0}, p_{1}, p_{2}, \ldots, p_{n} \in \mathbb{R}^{n}$ такой набор точек, что векторы $p_{1}-p_{0}, \ldots, p_{n}-p_{0}$ линейно независимы. Симплексом $\Delta$ с вершинами в этих точках мы назовем выпуклую оболочку множества $\left\{p_{0}, \ldots, p_{n}\right\}$. Пусть $d_{\Delta}$ обозначает диаметр симплекса $\Delta$.

Следует отметить, что использование треугольных и тетраэдральных сеток при численном решении задач математической физики весьма распространено. Для ознакомления можно обратиться к работам [1,2], а также к цитируемой в них литературе. Однако в указанных работах отсутствуют априорные оценки погрешности вычисления. Один из распространенных методов аппроксимации градиента функции заключается в следующем. Пусть функция $f(x) \in C^{1}\left(\mathbb{R}^{n}\right)$ и $\Delta-$ невырожденный симплекс. Тогда можно найти функцию вида $f_{\Delta}(x)=\langle a, x\rangle+b$ такую, что

$$
f\left(p_{i_{k}}\right)=f_{\Delta}\left(p_{i_{k}}\right), \quad k=0, \ldots, n .
$$

Существование и единственность такой функции следует из невырожденности симплекса $\Delta$. В качестве приближенного значения градиента $\nabla f(x)$ для точек $x \in \Delta$ можно считать значение градиента этой линейной функции $\nabla f_{\Delta}(x)$. Введем обозначение

$$
\delta_{\Delta}(f)=\max _{x \in \Delta}\left|\nabla f(x)-\nabla f_{\Delta}(x)\right|
$$

для погрешности вычисления градиента. Отметим, что оценки величины (1) имеются в работе J. R. Shewchuk [3], где, однако, рассматривается только случай $C^{2}$-гладких функций.

Пусть $A \subset \mathbb{R}^{n}$ - подмножество пространства $\mathbb{R}^{n}$. Множество $A$ называется дискретным, если оно замкнуто и все его точки изолированы. Из теоремы Больцано-Вейерштрасса следует, что такое множество локально конечно. Пусть $\varepsilon>0$. Дискретной $\varepsilon$-сетью $A$ в $\mathbb{R}^{n}$ назовем дискретное множество, являющееся $\varepsilon$-сетью. Последнее означает, что для всякой точки $x \in \mathbb{R}^{n}$ найдется точка $a \in A$, такая, что $|x-a|<\varepsilon$. В силу локальной конечности дискретной $\varepsilon$-сети можно рассмотреть ее триангуляцию $T$. Триангуляция $T$ представляет собой набор симплексов $\left\{\Delta_{k}\right\} k=1,2, \ldots$, которые имеют вершинами точки из $A$, попарно не пересекаются по внутренним точкам и объединение которых дает все пространство $\mathbb{R}^{n}$. Аппроксимация градиента функции $f(x) \in C^{1}\left(\mathbb{R}^{n}\right)$ осуществляется посредством аппроксимации его в каждой ячейке сети - симплексе $\Delta$ триангуляции - градиентом функции $f_{\Delta}(x)$.

Существует большое количество примеров, в том числе классический пример Шварца [4], иллюстрирующих тот факт, что поведение погрешности $\delta_{\Delta}(f)$ тесно связно со структурой симплекса (в двумерном случае треугольника), определяющей степень его невырожденности. Так, в работе [5] и частично в работе [6] было доказано, что при $n=2$ в случае триангуляции Делоне (см. [7]) набора точек $P$, образующих $\varepsilon$-сеть, справедлива оценка

$$
\max _{\Delta \in T} \delta_{\Delta}(f) \leq C(f) \varepsilon
$$

с константой $C(f)$, зависящей от класса функции $f$. Это неравенство является следствием неравенства для погрешности в отдельном треугольнике

$$
\delta_{\Delta}(f) \leq C(f) \cdot R(\Delta)
$$

где $R(\Delta)$ - радиус описанной окружности треугольника $\Delta$. Предпринятые попытки распространить эти результаты на случай многомерных пространств не привели к ожидаемому успеху. Тем не менее в работе [5] для случая $n=3$ было доказано неравенство, аналогичное неравенству (2) для триангуляций, в которой все тетраэдры являются остроугольными, т.е. у тетраэдров все плоские и двугранные углы являются острыми. Несмотря на это, вопрос о существовании оценки вида (3) до определенного момента времени оставался открытым. Существование такой оценки давало бы возможность использовать триангуляию Делоне в вопросах приближенного вычисления производных функций, заданных на нерегулярных сетках. Однако, как оказалось, при $n \geq 3$ оценку (3) невозможно получить принципиально. Соответствующий пример был построен в работе [8]. 
Вопрос о существовании оценки вида (2) для тетраэдральных сеток, удовлетворяющих условию Делоне, также был решен в этой работе. К сожалению, в отличие от плоских триангуляций, в многомерном случае для существования подобной оценки одного условия пустоты сферы недостаточно.

В настоящей статье мы вводим характеристику симплекса - коэффициент изопериметричности и с помощью нее получаем ряд оценок погрешности $\delta_{\Delta}(f)$. Ключевым результатом для этих оценок является получение оценки погрешности вычисления градиента в терминах погрешности вычисления самой функции на границе отдельного элемента разбиения и его коэффициента изопериметричности. Кроме того, мы даем оценки отклонения коэффициента изопериметричности при квазиизометричном преобразовании симплекса, которые могут быть полезны при контроле невырожденности ячеек расчетных сеток при их построении вариационными методами [9].

\section{1. ОЦЕНКА ПОГРЕШНОСТИ $\delta_{\Delta}(f)$}

Пусть $G$ - произвольная область в пространстве $\mathbb{R}^{n}$ с кусочно-гладкой границей. Пусть в области $G$ задана функция класса $C^{1}$ и пусть $\omega_{f}(t), t \geqslant 0$, обозначает модуль непрерывности ее градиента так, что

$$
|\nabla f(x)-\nabla f(y)| \leq \omega_{f}(|x-y|),
$$

для всех $x, y \in G$. Пусть имеется вторая функция подобного вида $g \in C^{1}(G)$, которая будет играть роль аппроксимирующей функции для функции $f$.

Теорема 1. Для всякой точки $x \in G$ имеет место неравенство

$$
|\nabla f(x)-\nabla g(x)| \leq \frac{1}{|G|}\left(\int_{G}\left(\omega_{f}(|x-y|)+\omega_{g}(|x-y|)\right) d y+\int_{\partial G}|f(y)-g(y)| d S\right) .
$$

Доказательство. Введем обозначение $\varphi(x)=f(x)-g(x)$. Тогда, очевидно, что

$$
\omega_{\varphi}(t) \leq \omega_{f}(t)+\omega_{g}(t)
$$

для всех $t \geq 0$. Для произвольного векторного поля $X \in C^{1}(G) \cap C(\bar{G})$ согласно формуле ГауссаОстроградского имеем:

$$
\int_{G}\langle\nabla \varphi(y), X\rangle d y=-\int_{G} \varphi(y) \operatorname{div} X d y+\int_{\partial G} \varphi(y)\langle X, \nu\rangle d S,
$$

где $\nu$ - вектор внешней нормали к границе области $G$. Зафиксируем точку $x \in G$ и положим в равенстве выше $X=\nabla \varphi(x)$. Получим:

$$
\int_{G}\langle\nabla \varphi(y), \nabla \varphi(x)\rangle d y=\int_{\partial G} \varphi(y)\langle\nabla \varphi(x), \nu\rangle d S,
$$

или

$$
\int_{G}\langle\nabla \varphi(x), \nabla \varphi(x)\rangle d y=\int_{G}\langle\nabla \varphi(x)-\nabla \varphi(y), \nabla \varphi(x)\rangle d y+\int_{\partial G} \varphi(y)\langle\nabla \varphi(x), \nu\rangle d S .
$$

Учитывая, что $|\nabla \varphi(x)-\nabla \varphi(y)| \leq \omega_{\varphi}(|x-y|)$, получаем:

$$
|G||\nabla \varphi(x)| \leq \int_{G} \omega_{\varphi}(|x-y|) d y+\int_{\partial G}|\varphi(y)| d S .
$$

И - окончательно

$$
|\nabla f(x)-\nabla g(x)| \leq \frac{1}{|G|}\left(\int_{G}\left(\omega_{f}(|x-y|)+\omega_{g}(|x-y|)\right) d y+\int_{\partial G}|f(y)-g(y)| d S\right) .
$$

Что и требовалось доказать. 
Замечание. Доказанное неравенство связывает погрешность вычисления градиента функции внутри области через аппроксимацию самой функции на границе.

Отношение

$$
\sigma(G)=\frac{|\partial G|^{\frac{n}{n-1}}}{|G|}
$$

мы назовем коэффициентом изопериметричности области $G$. Доказанное в теореме неравенство можно переписать в виде

$$
|\nabla f(x)-\nabla g(x)| \leq \frac{1}{|G|} \int_{G}\left(\omega_{f}(|x-y|)+\omega_{g}(|x-y|)\right) d y+\sigma(G) \frac{\sup _{x \in \partial G}|f(y)-g(y)|}{|\partial G|^{1 /(n-1)}} .
$$

Выберем в качестве области $G$ невырожденный симплекс с вершинами в точках $p_{0}, p_{1}, \ldots, p_{n} \in \mathbb{R}^{n}$. Величина $\sigma(\Delta)$ симплекса $\Delta$ отвечает за отклонение произвольного симплекса от правильного, поскольку, как хорошо известно, эта величина достигает своего минимального значения для правильного симплекса. Из доказанной теоремы следует оценка погрешности, если в качестве приближающей функции $g(x)$ выбрать аффинную функцию $f_{\Delta}(x)$, совпадающую с функцией $f(x)$ в вершинах симплекса.

Теорема 2. Пусть функция $f \in C^{1}(\Delta)$ u $\omega_{f}(t)$ - модуль непрерывности ее градиента. Тогда

$$
\delta_{\Delta}(f)=\sup _{x \in \Delta}\left|\nabla f(x)-\nabla f_{\Delta}(x)\right| \leq \omega_{f}(\operatorname{diam}(\Delta))+\sigma(\Delta) \frac{\sup _{x \in \partial \Delta}\left|f(x)-f_{\Delta}(x)\right|}{|\partial \Delta|^{1 /(n-1)}} .
$$

Лемма 1. Пусть имеется невырожденный симплекс $\Delta$ с вершинами в точках $p_{0}, p_{1}, \ldots$, $p_{n} \in \mathbb{R}^{n}$. Предположим, что функция $f \in C^{1}(\Delta)$ такая, что $f\left(p_{i}\right)=0$ для всякого $i=0,1, \ldots, n$. Тогда имеет место неравенство

$$
\max _{x \in \Delta}|f(x)| \leq \int_{0}^{R} \omega_{f}(t) d t
$$

где $\omega_{f}(t)$ - модуль непрерывности градиента функции $f(x)$, а $R$ - радиус описанной сферы симплекса $\Delta$.

Доказательство. Рассмотрим сначала случай $n=1$. Из условия леммы следует, что найдется точка $p \in\left[p_{0}, p_{1}\right]$, в которой функция $f(x)$ принимает максимальное значение. Тогда $f^{\prime}(p)=0$. Следовательно,

$$
\begin{aligned}
f(p)-f\left(p_{0}\right)= & \int_{0}^{1} \frac{d}{d t} f\left(p+t\left(p-p_{0}\right)\right) d t=\int_{0}^{1} f^{\prime}\left(p+t\left(p-p_{0}\right)\right)\left(p-p_{0}\right) d t= \\
& =\int_{0}^{1}\left(f^{\prime}\left(p+t\left(p-p_{0}\right)\right)-f^{\prime}(p)\right)\left(p-p_{0}\right) d t .
\end{aligned}
$$

По определению модуля непрерывности имеем

$$
|f(p)| \leq \int_{0}^{1} \omega_{f}\left(t\left|p-p_{0}\right|\right)\left|p-p_{0}\right| d t=\int_{0}^{\left|p-p_{0}\right|} \omega_{f}(t) d t .
$$

Откуда получаем оценку

$$
\max _{\left[p_{0}, p_{1}\right]}|f(x)| \leq \int_{0}^{\left|p_{1}-p_{0}\right| / 2} \omega_{f}(t) d t=\int_{0}^{R} \omega_{f}(t) d t
$$


Пусть теперь $n>1$. Предположим, что максимум функции $|f(x)|$ достигается во внутренней точке $p \in \Delta$. Выбирая вершину $p_{k}$, ближайшую к этой точке, как и выше, получим:

$$
\begin{aligned}
f(p)-f\left(p_{k}\right)= & \int_{0}^{1} \frac{d}{d t} f\left(p+t\left(p-p_{k}\right)\right) d t=\int_{0}^{1}\left\langle\nabla f\left(p+t\left(p-p_{k}\right)\right), p-p_{k}\right\rangle d t= \\
& =\int_{0}^{1}\left\langle\nabla f\left(p+t\left(p-p_{k}\right)\right)-\nabla f(p), p-p_{k}\right\rangle d t .
\end{aligned}
$$

По определению модуля непрерывности имеем:

$$
|f(p)| \leq \int_{0}^{1} \omega_{f}\left(t\left|p-p_{k}\right|\right)\left|p-p_{k}\right| d t=\int_{0}^{\left|p-p_{k}\right|} \omega_{f}(t) d t .
$$

Поскольку $\left|p-p_{k}\right| \leq R$, получаем оценку

$$
\max _{\Delta}|f(x)| \leq \int_{0}^{R} \omega_{f}(t) d t
$$

Если точка максимума модуля функции лежит на одной из граней симплекса, то аналогичные рассуждения мы проводим на $(n-1)$-мерной плоскости этой грани. Применяя тот факт, что радиус описанной сферы для грани не превосходит радиуса описанной сферы симплекса, получим требуемое неравенство. Таким образом, мы можем поступать пока не придем к случаю, когда точка максимума лежит на одном из ребер симплекса. А этот случай уже раньше был рассмотрен. Лемма доказана.

Следствие 1. Пусть функция $f \in C^{1}(\Delta) u \omega_{f}(t)$ - модуль непрерыьвности ее градиента. Тогда

$$
\delta_{\Delta}(f)=\sup _{x \in \Delta}\left|\nabla f(x)-\nabla f_{\Delta}(x)\right| \leq \omega_{f}(\operatorname{diam}(\Delta))+\sigma(\Delta) \frac{1}{|\partial \Delta|^{1 /(n-1)}} \int_{0}^{R} \omega_{f}(t) d t .
$$

Следствие 2. Пусть функция $f \in C^{1, \alpha}(\Delta), 0<\alpha \leq 1$, т.е. найдется постоянная $M>0$ такая, что для всех $x, y \in S$ выполнено

$$
|\nabla f(x)-\nabla f(y)| \leq M|x-y|^{\alpha} .
$$

Тогда

$$
\delta_{\Delta}(f)=\sup _{x \in \Delta}\left|\nabla f(x)-\nabla f_{\Delta}(x)\right| \leq M \operatorname{diam}(\Delta)^{\alpha}+\sigma(\Delta) \frac{1}{(\alpha+1)|\partial \Delta|^{1 /(n-1)}} R^{\alpha+1} .
$$

Лемма 2. Пусть $\Delta$ - невырожденный симплекс с вершинами в точках $p_{0}, p_{1}, \ldots, p_{n}$. Если $r=r(\Delta)-$ радиус вписанной сферы симплекса $\Delta$, то

$$
\frac{|\partial \Delta|}{|\Delta|}=\frac{n !}{r(\Delta)}
$$

Доказательство. несложно и обобщает известное соотношение из элементарной геометрии.

Теорема 3. Пусть функция $f \in C^{1}(\Delta)$ и $\omega_{f}-$ модуль непрерывности ее градиента. Тогда имеет место оценка погрешности

$$
\delta_{\Delta}(f) \leq \omega_{f}(\operatorname{diam}(\Delta))+\frac{n !}{r} \int_{0}^{R} \omega_{f}(t) d t .
$$

Напомним (см. например [6]), что триангуляция $T$ данного набора точек $P \subset \mathbb{R}^{n}$ называется триангуляцией Делоне, если описанная сфера каждого симплекса из $T$ не содержит внутри себя точек из $P$. В этом случае также говорят, что для таких сфер выполнено условие пустого шара. 
Теорема 4. Пусть Т - триангуляция Делоне дискретной в-сети А. Тогда радиус описанной сферы каждого симплекса триангуляции не превосходит $\varepsilon$.

Доказательство. Предположим, что теорема не верна. Найдется симплекс $S \in T$, у которого радиус описанной сферы больше $\varepsilon$. Пусть $x_{0}$ - центр этой сферы. Рассмотрим шар с центром в точке $x_{0}$ и радиуса $\varepsilon$. В силу условия Делоне этот шар не может содержать вершин триангуляции, т.е. точек множества $A$. Но это противоречит определению $\varepsilon$-сети. Теорема доказана.

Пусть имеется дискретная $\varepsilon$-сеть и ее триангуляция Делоне $T$. Предположим, что величина отношения радиуса описанной сферы к радиусу вписанной ограничена

$$
\mu(T)=\sup _{S \in T} \frac{R(S)}{r(S)}<+\infty .
$$

Следующая теорема получается как следствие оценок из теоремы 3 и теоремы 4.

Теорема 5. Пусть функция $f \in C^{1}\left(\mathbb{R}^{n}\right)$ с равномерно непрерывным градиентом, и $\omega_{f}(t)-$ модуль непрерывности ее градиента. Тогда для любого симплекса $S \in T$ имеет место оценка погрешности

$$
\delta_{S}(f) \leq \omega_{f}(2 \varepsilon)+n ! \frac{\mu(T)}{\varepsilon} \int_{0}^{\varepsilon} \omega_{f}(t) d t .
$$

Следствие 3. Пусть функция $f \in C^{1, \alpha}\left(\mathbb{R}^{n}\right), 0<\alpha \leq 1$. Тогда для любого симплекса $S \in T$ имеет место оценка погрешности

$$
\delta_{S}(f) \leq M\left(2^{\alpha}+n ! \frac{\mu(T)}{\alpha+1}\right) \varepsilon^{\alpha} .
$$

\section{2. ИСКАЖЕНИЕ КОЭФФИЦИЕНТА ИЗОПЕРИМЕТРИЧНОСТИ ПРИ КВАЗИИЗОМЕТРИИ}

Следуя введенной терминологии, коэффициент изопериметричности $n$-мерного симплекса $\Delta$ равен

$$
\sigma(\Delta)=\frac{|\partial \Delta|^{n /(n-1)}}{|\Delta|} .
$$

Величина $\sigma(\Delta)$ характеризует отклонение произвольного симплекса $\Delta$ от правильного, поскольку минимальное значение достигается на правильном симплексе. Изучим поведение этой характеристики симплекса при квазиизометричном преобразовании. Напомним, что отображение $F: \mathbb{R}^{n} \rightarrow \mathbb{R}^{n}$ называется квазиизометричным, если найдутся постоянные $0<l \leq L<+\infty$ такие, что для любой пары точек $x, y \in \mathbb{R}^{n}$ выполнено

$$
l \cdot|x-y| \leq|F(x)-F(y)| \leq L \cdot|x-y| .
$$

Прежде всего докажем ряд вспомогательных утверждений.

Пусть $\left\{d_{i j}: 0 \leqslant i<j \leqslant n\right\}-$ совокупность $n(n+1) / 2$ переменных. Рассмотрим квадратную $(n+2) \times(n+2)$ матрицу [10]

$$
C M_{n}:=\left\|c_{i j}\right\|= \begin{cases}0, & i=j, \\ 1, & i=1, \quad j=2, \ldots, n+2, \\ 1, & j=1, \quad i=2, \ldots, n+2, \\ d_{i-2 j-2}, & i \neq j, \quad 2 \leq i, \quad j \leq n+2 .\end{cases}
$$

Многочлен $\Gamma_{n}:=\operatorname{det}\left(C M_{n}\right) \in \mathbb{Z}$ от многих переменных $d_{i j}, 0 \leqslant i<j \leqslant n$, называется определителем Кэли - Менгера. Этот определитель дает формулу для вычисления $n$-мерного объема $V$ симплекса $T$ в терминах евклидовых расстояний $\left\{d_{i j}=\operatorname{dist}\left(v_{i}, v_{j}\right): 0 \leqslant i<j \leqslant n\right\}$ между рассматриваемыми точками:

$$
V^{2}=\frac{(-1)^{n+1}}{2^{n}(n !)^{2}} \Gamma_{n}\left(d_{01}, d_{02}, \ldots, d_{(n-1) n}\right) .
$$


Лемма 3. Пусть $A_{n}-$ квадратная матрица n-го порядка с неотрицательньми коэффициентами, содержащая ровно п нулевых элементов так, что никакие два из них не принадлежат

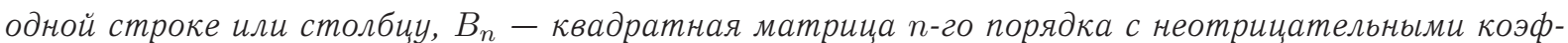
фициентами, содержащая ровно $n-1$ нулевых элементов, никакие два из которых не принадлежат одной строке или столбиу. Каждое из n! слагаемьх в разложении определителей этих матриц представляет собой однородный одночлен с нулевым, отрицательным или положительньм коэффициетом. функция $g: M_{n} \rightarrow \mathbb{N}$ сопоставляет квадратной матрице $M_{n}$ количество нулевых слагаемых в выражении определителя, тогда значения функции $g\left(A_{n}\right)$ при $n \geq 4$ можно вычислить по рекуррентным формулам

$$
g\left(A_{n}\right)=(n-1) !+(n-1) g\left(B_{n-1}\right)
$$

где $g\left(B_{n-1}\right)=g\left(A_{n-2}\right)+(n-2) g\left(B_{n-2}\right)$ u $g\left(A_{2}\right)=g\left(B_{2}\right)=1, g\left(A_{3}\right)=4$.

Доказательство. Ясно, что значение $g\left(A_{n}\right)$ не зависит от положительных коэффициентов матрицы и поэтому достаточно рассмотреть матрицу $A_{n}$, состоящую из нулей и единиц

$$
C_{n}=\left\|c_{i j}\right\|= \begin{cases}0, & i=j \\ 1, & i \neq j\end{cases}
$$

Разложив определитель этой матрицы по элементам одной из строк, например первой, получим:

$$
\operatorname{det} C_{n}=0 \cdot \underbrace{\operatorname{det} C_{n-1}}_{(n-1) ! \text { слагаемых }}+\sum_{i=2}^{n}(-1)^{1+i} \operatorname{det} C_{n-1}^{(i)},
$$

где $C_{n-1}^{(i)}-$ матрица размерности $n-1$, отличающаяся от $C_{n-1}$ тем, что по главной диагонали на $i$-м месте стоит 1 , т. е. это матрица вида $B_{n-1}$. Отсюда следует первое равенство. Расписав определитель матрицы $C_{n-1}^{(i)}$ по элементам одной из строк получим второе равенство утверждения.

Для многочлена объема симплекса справедливо

Следствие 4. В многочлене объема количество нулевых слагаемых равно $g\left(C M_{n}\right)=g\left(A_{n+2}\right)$, положительных слагаемых $-p_{n}=0.5 \cdot\left((n+2) !-g\left(C M_{n}\right)+(n+1)\right.$, а отрицательных $q_{n}=0.5 \cdot\left((n+2) !-g\left(C M_{n}\right)-(n+1)\right)$.

Доказательство. Найдем разницу количества положительных и отрицательных слагаемых в выражении объема, для этого, как и при доказательстве леммы 3 , рассмотрим матрицу $C_{k}$, состоящую из нулей и единиц. Докажем, что определитель этой матрицы $\operatorname{det} C_{k}=(-1)^{k-1}(k-1)$. Это будет означать, что количество положительных и отрицательных слагаемых в выражении определителя матрицы вида $A_{n}$ отличается на $n-1$ (соответственно на $n+1$ для $C M_{n}$ ), а знаки «+» или «-» указывают на то, каких слагаемых в выражении больше, положительных или отрицательных. Доказательство проведем методом математической индукции. Равенство $\operatorname{det} C_{2}=-1$ очевидно.

Предположим, что $\operatorname{det} C_{k-1}=(-1)^{k-2}(k-2)$, вычислим $\operatorname{det} C_{k}$. Для этого вычтем первый столбец матрицы из остальных и полученную матрицу разложим по элементам второй строки:

$$
\operatorname{det} C_{k}=(-1)^{k-1}-\operatorname{det} C_{k-1}=(-1)^{k-1}+(-1)^{k-1}(k-2)=(-1)^{k-1}(k-1) \text {. }
$$

С учетом формулы (1) получим требуемое.

Теорема 6. Пусть в пространстве $\mathbb{R}^{n}$ задан невырожденный симплекс $\Delta$, максимальное расстояние между вершинами которого равно $d$, минимальное - a, а наименьшая площадь $(n-1)$-мерной грани равна $S$, и квазиизометрическое отображение $F: \mathbb{R}^{n} \rightarrow \mathbb{R}^{n}$ с константами l, L такими, ито

$$
\frac{L}{l}=k<\sqrt[2 n]{1+\frac{2 n a^{2 n-2} d^{2}-(n-1) a^{2 n}}{q_{n} d^{2 n}}}
$$


Тогда для коэффициента изопериметричности образа симплекса справедлива оценка

$$
\frac{\sigma}{k^{n}} \frac{\sqrt[2 n-2]{\left(1-\left(k^{2 n-2}-1\right) \mu_{1}(\Delta)\right)^{n}}}{\sqrt{1+\left(1-k^{-2 n}\right) \mu_{2}(\Delta)}} \leqslant \sigma^{\prime} \leqslant \sigma k^{n} \frac{\sqrt[2(n-1)]{\left(1+\left(1-k^{2-2 n}\right) \mu_{1}(\Delta)\right)^{n}}}{\sqrt{1-\left(k^{2 n}-1\right) \mu_{2}(\Delta)}},
$$

где $r_{n}=2^{n}(n !)^{2}, \mu_{1}(\Delta)=q_{n-1} d^{2(n-1)} /\left(r_{n-1} S^{2}\right), \mu_{2}(\Delta)=q_{n} d^{2 n} /\left(r_{n} V^{2}\right)$.

Доказательство. Представим выражение для объема образа симплекса в виде

$$
V^{\prime 2}=\frac{1}{2^{n}(n !)^{2}}\left(P^{\prime}-Q^{\prime}\right),
$$

где $P^{\prime}$ и $-Q^{\prime}-$ суммы всех слагаемых, которые входят в многочлен $n$-мерного объема симплекса со знаком «+» и «-» соответственно.

Поскольку $P^{\prime}$ и $Q^{\prime}$ связаны с соответствующими величинами прообраза неравенствами $l^{2 n} P \leqslant P^{\prime} \leqslant L^{2 n} P$ и $-L^{2 n} Q \leqslant-Q^{\prime} \leqslant-l^{2 n} Q$, получаем:

$$
l^{2 n} P-L^{2 n} Q \leqslant 2^{n}(n !)^{2} V^{\prime 2} \leqslant L^{2 n} P-l^{2 n} Q,
$$

откуда

$$
l^{2 n} 2^{n}(n !)^{2} V^{2}-\left(L^{2 n}-l^{2 n}\right) Q \leqslant 2^{n}(n !)^{2} V^{\prime 2} \leqslant L^{2 n} 2^{n}(n !)^{2} V^{2}+\left(L^{2 n}-l^{2 n}\right) Q .
$$

В свою очередь, $Q \leqslant q_{n} d^{2 n}$, где $q_{n}-$ количество отрицательных слагаемых в многочлене объема, а $d-$ наибольшее расстояние между вершинами исходного симплекса, следовательно, объем полученного симплекса

$$
l^{n} V \sqrt{1-\frac{L^{2 n}-l^{2 n}}{l^{2 n}} \frac{q_{n} d^{2 n}}{2^{n}(n !)^{2} V^{2}}} \leqslant V^{\prime} \leqslant L^{n} V \sqrt{1+\frac{L^{2 n}-l^{2 n}}{L^{2 n}} \frac{q_{n} d^{2 n}}{2^{n}(n !)^{2} V^{2}}} .
$$

Аналогично находим площади граней симплекса как $(n-1)$-мерные объемы:

$$
\begin{gathered}
l^{n-1} S_{i} \sqrt{1-\frac{L^{2 n-2}-l^{2 n-2}}{l^{2 n-2}} \frac{q_{n-1} d_{i}^{2 n-2}}{2^{n-1}((n-1) !)^{2} S_{i}^{2}}} \leqslant S_{i}^{\prime} \leqslant \\
\leqslant L^{n-1} S_{i} \sqrt{1+\frac{L^{2 n-2}-l^{2 n-2}}{L^{2 n-2}} \frac{q_{n-1} d_{i}^{2 n-2}}{2^{n-1}((n-1) !)^{2} S_{i}^{2}}},
\end{gathered}
$$

где $i=0,1, \ldots, n$, а $d_{i}-$ наибольшее расстояние между вершинами $i$-й грани. Обозначим через $S=\min S_{i}, i=0,1, \ldots, n$, а так как $d=\max d_{i}$, то суммируя все оценки площадей, получим, что для площади полной поверхности образа симплекса справедливо

$$
\begin{gathered}
l^{n-1}|\partial \Delta| \sqrt{1-\frac{L^{2 n-2}-l^{2 n-2}}{l^{2 n-2}} \frac{q_{n-1} d^{2 n-2}}{2^{n-1}((n-1) !)^{2} S^{2}}} \leqslant\left|\partial \Delta^{\prime}\right| \leqslant \\
\leqslant L^{n-1}|\partial \Delta| \sqrt{1+\frac{L^{2 n-2}-l^{2 n-2}}{L^{2 n-2}} \frac{q_{n-1} d^{2 n-2}}{2^{n-1}((n-1) !)^{2} S^{2}}} .
\end{gathered}
$$

Применив формулу коэффициента изопериметричности к полученным оценкам и переобозначив коэффициенты, получим неравенство теоремы.

В случае пространства $\mathbb{R}^{2}$ для треугольников справедливо

Следствие 5. Пусть на плоскости заданы треугольник $\Delta$, у которого а и с - длинь максимальной и минимальной стороны соответственно, и квазиизометрическое отображение $F: \mathbb{R}^{2} \rightarrow \mathbb{R}^{2}$ с константами $k=\frac{L}{l}<\sqrt[4]{1+\frac{2 c^{2}}{3 a^{2}}}$, тогда для коэффициента изопериметричности образа треугольника справедлива оценка

$$
k^{-2} \frac{\sigma}{\sqrt{1+\left(1-k^{-4}\right) \mu(\Delta)}} \leqslant \sigma^{\prime} \leqslant k^{2} \frac{\sigma}{\sqrt{1-\left(k^{4}-1\right) \mu(\Delta)}},
$$

где $\mu(\Delta)=3 a^{4}(4 S)^{-2}$. 
В этом случае легко получить оценку только через коэффицент изопериметричности исходного треугольника

Следствие 6. Пусть на плоскости задань треугольник $\Delta$, у которого а и с - длинь максимальной и минимальной сторонь соответственно, и квазиизометрическое отображение $F: \mathbb{R}^{2} \rightarrow \mathbb{R}^{2}$ с константами $k=\frac{L}{l}<\sqrt[4]{1+\frac{2 c^{2}}{3 a^{2}}}$, тогда для коэффициента изопериметричности образа треугольника справедлива оценка

$$
k^{-2} \frac{\sigma}{\sqrt{1+\left(1-k^{-4}\right)(4 \sigma)^{-2}}} \leqslant \sigma^{\prime} \leqslant k^{2} \frac{\sigma}{\sqrt{1-\left(k^{4}-1\right)(4 \sigma)^{-2}}} .
$$

Работа выполнена при финансовой поддержке РФФИ (проект № 15-41-02517).

\section{Библиографичческий список}

1. Боровиков С. Н., Иванов И. Э., Крюков И. А. Моделирование пространственных течений идеального газа с использованием тетраэдральных сеток // Матем. моделирование. 2006. Т. 18, № 8. C. $37-48$.

2. Боровиков С. Н., Крюков И. А., Иванов И. Э. Построение нерегулярных треугольных сеток на криволинейных гранях на основе триангуляции Делоне // Матем. моделирование. 2005. Т. 17, № 8. C. 31-45.

3. Shewchuk J. R. What is a Good Linear Element? Interpolation, Conditioning and Quality Measures. Preprint / Department of Electrical Engineering and Computer Sciences University of California at Berkeley. Berkeley, 2002. CA 94720.

4. Гелбаум Б., Олмстед Дж. Контрпримеры в анализе. Волгоград : Изд-во ПЛАТОН, 1997.

5. Клячин B. А., Широкий А. А. Триангуляция Делоне многомерных поверхностей и ее аппрокси- мационные свойства // Изв. вузов. Математика. 2012. № 1. С. 31-39.

6. Клячин B. A., Пабат E. A. $C^{1}$-аппроксимация поверхностей уровня функций, заданных на нерегулярных сетках // Сиб. журн. индустр. матем. 2010. T. 13, № 2. С. 69-78.

7. Сквориов А. В., Мирза Н. С. Алгоритмы построения и анализа триангуляции. Томск : Изд-во Томск. ун-та, 2006.

8. Клячин B. А. О многомерном аналоге примера Шварца // Изв. РАН. Сер. матем. 2012. Т. 76, № 4. С. 41-48.

9. Ушакова О. В. Условия невырожденности трехмерных ячеек. Формула для объема ячеек // Журн. вычисл. матем. и матем. физ. 2001. Т. 41, № 6. С. 881-894.

10. Берже М. Геометрия : в 2 т. М. : Мир, 1984. Т. 1. $560 \mathrm{c}$.

\section{Isoperimetry Coefficient for Simplex in the Problem of Approximation of Derivatives}

\section{A. Klyachin, D. V. Shurkaeva}

Volgograd State University, 100, Universitetsky prospekt, 400062, Volgograd, Russia, klchnv@mail.ru, diana-547@ya.ru

We introduce the isoperimetry coefficient $\sigma(G)=|\partial G|^{n /(n-1)} /|G|$ of region $G \subset \mathbb{R}^{n}$. In terms of this the error $\delta_{\Delta}(f)$ estimates for the gradient of the piecewise linear interpolation of functions of class $C^{1}(G), C^{2}(G), C^{1, \alpha}(G), 0<\alpha<1$, are obtained. The problem of obtaining such estimates is nontrivial, especially in the multidimensional case. Here it should be noted that in the two-dimensional case, for functions of class $C^{2}(G)$, the convergence of the derivatives is provided by the classical Delaunay condition. In the multidimensional case, as shown by the examples, such conditions are not sufficient. Nevertheless, the article shows how to apply these estimates to the Delaunay triangulation of multidimensional discrete $\varepsilon$-nets. The results obtained give sufficient conditions for convergence of the derivatives on the Delaunay triangulation of discrete $\varepsilon$-nets with $\varepsilon \rightarrow 0$. In addition, the ratio of the distortion factor is found for isoperimetry coefficient under the quasi-isometric transformation.

Key words: isoperimetry coefficient, simplex, piecewise linear interpolation.

This work was supported by the Russian Foundation for Basic Research (projects no. 15-41-02517).

\section{References}

1. Borovikov S. N., Ivanov I. E., Kryukov I. A. Modeling of test ideal gas dynamics problems using tetrahedral meshes. Matem. Mod., 2006, vol. 18, no. 8, pp. 37-48 (in Russian). 
2. Borovikov S. N., Kryukov I. A., Ivanov I. E. Unstructured triangular mesh generation on curved faces based on Delauney triangulation. Matem. Mod., 2005, vol. 17, no. 8, pp. 31-45 (in Russian).

3. Shewchuk J. R. What is a Good Linear Element? Interpolation, Conditioning and Quality Measures. Preprint / Department of Electrical Engineering and Computer Sciences University of California at Berkeley, Berkeley, 2002, CA 94720.

4. Gelbaum B. R., Olmsted J. M. H. Counterexamples in analysis. San Francisco, London, Amsterdam, Holden-Day, 1964.

5. Klyachin V. A., Shirokii A. A. The Delaunay triangulation for multidimensional surfaces and its approximative properties. Russian Math. [Izvestiya VUZ. Matematika], 2012, vol. 56, no. 1, pp. 27-34. DOI: $10.3103 /$ S1066369X12010045.

6. Klyachin V. A., Pabat E. A. $C^{1}$-approximation of the level surfaces of functions defined on irregular grids. Sib. Zh. Ind. Mat., 2010, vol. 13, no. 2, pp. 69-78 (in Russian).

7. Skvortsov A. V., Mirza N. S. Algoritmy postroeniya $i$ analiza triangulacii [Constructing and Analisys of Triangulation Algorithms] Tomsk, Tomsk Univ. Press, 2006 (in Russian).

8. Klyachin V. A. On a multidimensional analogue of the Schwarz example. Izv. Math., 2012, vol. 76 , no. 4 , pp. 681-687. DOI: 10.1070/IM2012 v076n04ABEH002601.

9. Ushakova O. V. Nondegeneracy conditions for three-dimensional cells and a formula for the cell's volume. Comput. Math. Math. Phys., 2001, vol. 41, no. 6 , pp. 832-845.

10. Berger M. Geometry Translated from the French by $M$. Cole and S. Levy. Universitext. Berlin, Springer-Verlag, 1987. xiv+428 p.

\section{ASYMPTOTIC RATIO OF HARMONIC MEASURES OF SLIT SIDES}

\section{V. Prokhorov, D. V. Ukrainskii}

Saratov State University, 83, Astrakhanskaya st., 410012, Saratov, Russia, ProkhorovDV @ info.sgu.ru, D.V.Ukrainskiy@gmail.com

The article is devoted to the geometry of solutions to the chordal Löwner equation which is based on the comparison of singular solutions and harmonic measures for the sides of a slit in the upper half-plane generated by a driving term. An asymptotic ratio for harmonic measures of slit sides is found for a slit which is tangential to a straight line under a given angle, and for a slit with high order tangency to a circular arc tangential to the real axis.

Key words: Löwner equation, singular solution, harmonic measure, half-plane capacity.

\section{INTRODUCTION}

The famous Löwner differential equation have been introduced in 1923 [1] and was aimed to give a parametric representation of slit domains. In this article we describe an asymptotic behavior of singular solutions and harmonic measures for the sides of a slit in domains generated by a driving term of the Löwner equation.

The chordal version of the Löwner equation deals with the upper half-plane $\mathbb{H}=\{z: \operatorname{Im} z>0\}$, $\mathbb{R}=\partial \mathbb{H}$, and functions $f(z, t)$ normalized near infinity by

$$
f(z, t)=z+\frac{2 t}{z}+O\left(\frac{1}{z^{2}}\right)
$$

which solve the chordal Löwner differential equation

$$
\frac{d f(z, t)}{d t}=\frac{2}{f(z, t)-\lambda(t)}, \quad f(z, 0) \equiv z, \quad t \geq 0,
$$

and map subdomains of $\mathbb{H}$ onto $\mathbb{H}$. Here $\lambda(t)$ is a real-valued continuous driving term.

Let $\gamma_{t}:=\gamma[0, t]=\{\gamma(x): 0 \leq x \leq t\}$ be a simple continuous curve in $\mathbb{H} \cup\{0\}$ with endpoints $\gamma(0)=0$ and $\gamma(t), 0 \leq t \leq T$. Then there is a unique map $f(z, t): \mathbb{H} \backslash \gamma_{t} \rightarrow \mathbb{H}$ satisfying the chordal Löwner equation (1) with $\lambda(t)$ uniquely determined by $\gamma[0, t]$. The function $f(z, t)$ can be extended continuously to $\mathbb{R} \cup \gamma(t)$, and $f(\gamma(t), t)=\lambda(t)$. The value $t$ is called the half-plane capacity of the curve $\gamma_{t}, t=h \operatorname{cap}\left(\gamma_{t}\right)$, see, e.g. [2].

We say that $\gamma_{t} \in C^{n}, n \in \mathbb{N}$, on $[0, S]$ if, for the arc-length parameter $s$ of $\gamma_{t}, \gamma(t(s))$ has a continuous derivative $\gamma^{(n)}$ in $s$ on $[0, S], t(S)=T$. All the derivatives $\gamma^{(k)}, 1 \leq k \leq n$, at $s=0$ are understood as one-side derivatives. A curve $\gamma_{t} \in C^{n}, \gamma(0)=0$, is said to have at least $n$-order tangency with a ray $I_{\theta}=\left\{e^{i \theta} s: s \geq 0\right\}, \theta \in \mathbb{R}$, at $s=0$ if

$$
\gamma(t(s))=e^{i \theta} s+o\left(s^{n}\right), \quad s \rightarrow+0 .
$$

